\title{
The Risk in the Insurance Field: A Generalized Analysis
}

\author{
Rosa Ferrentino $^{1^{*}}$ (D) Luca Vota ${ }^{2}$ \\ ${ }^{1}$ Department of Economic and Statistics Sciences, University of Salerno, Fisciano (Sa), Italy \\ ${ }^{2}$ Economics and Finance Scholar, University of Salerno, Fisciano (Sa), Italy \\ Email: ^rferrentino@unisa.it, votaluca@yahoo.it
}

How to cite this paper: Ferrentino, R. and Vota, L. (2020) The Risk in the Insurance Field: A Generalized Analysis. Journal of Mathematical Finance, 10, 200-221. https://doi.org/10.4236/jmf.2020.101013

Received: January 23, 2020

Accepted: February 25, 2020

Published: February 28, 2020

Copyright $\odot 2020$ by author(s) and Scientific Research Publishing Inc. This work is licensed under the Creative Commons Attribution-NonCommercial International License (CC BY-NC 4.0). http://creativecommons.org/licenses/by-nc/4.0/

Open Access

\begin{abstract}
The current developments in the insurance system and the innovation in the financial sector have highlighted a series of problems connected to the measurement of the risk and the assessment of the global risk has taken on particular importance. The literature on this interesting topic has led to a new approach that allows to decompose the sources of uncertainty that feed the global risk, to understand the importance of each source and to determine its contribution. This article presents an innovative method to calculate some statistical measures, to quantify the relative importance of each source of uncertainty and to allow analysts, therefore, a certain degree of control over the insurance system. To this end, in the paper, some tools are used and analyzed in detail for the assessment of the financial risk related to the management of a portfolio of life insurances policies such as the coefficient of determination and the risk index of the portfolio, with particular regard to the contribution offered by the pooling risk. In addition, it is shown a measurement of the Arrow-Pratt risk premium, with respect to the demographic risk and the overall market risk, proving that for a risk averse insurer with a certain wealth the two values are in direct proportionality relation.
\end{abstract}

\section{Keywords}

Risk Decomposition, Risk Pooling, Demographic Risk, Coefficient of Determination, Standard Deviation, Risk Index

\section{Introduction}

In the economic-financial context in which banks, companies and financial intermediaries currently operate, crises and corporate restructuring have placed great attention on the problem of the risk assessment, control and management, 
where the term risk is used to indicate both an economically unfavorable eventuality and an economically advantageous eventuality. The first meaning generally has a prevalence over the second. In other words, the risk is usually defined as the financial consequence of an uncertain event that can result in losses or in damages for the companies and the people involved.

In fact, the activity of most companies involves exposure to various types of risks, which if on the one hand are the prerequisite to achieve the set objectives and to obtain advantageous performances, on the other hand imply the danger of having face more or less predictable adverse situations; consequently, it is necessary to process and to develop risk management policies and to identify the solutions to provide to the market to reduce the impact that potential damage may have on corporate profits and on the survival of the company.

Since risk is an essential component in every economic system, it is necessary to adopt behaviors that allow us to guard against events that, although unlikely, could cause serious consequences. The first step is to identify the risk and to make an assessment; the second consists, instead, in avoiding it or controlling it or transferring it to another subject.

In other words, there are three ways to manage risk: avoiding, controlling or transferring it. Through the third way the risk of which an individual does not want to take charge is transferred to a third party. The third party that assumes the risk is the insurance company that undertakes, upon payment of a premium, to compensate the damages suffered against the occurrence of the event provided for in the insurance contract. In other words, to remove the negative consequences associated with a random event, it is possible to take out an insurance policy by which the risk, subject to the occurrence of a given event, is transferred to another person or to an insurance company. More precisely, the insured, instead of taking on the losses, can transfer the risk to a specialized company or a third economic entity; transferring the risk can thus minimize or eliminate the possible harmful consequences of the claims on the economic and on the financial equilibrium.

Obviously, insuring against a certain event does not imply avoiding its occurrence, but rather protecting from the negative effects related to it and this one translates into the supply, by the insurer, of asset guarantees for the insured, through future and possible performance. The possibility to survive can be examined mainly from two perspectives. The first one focuses on the company's overall exposure to the sources of risk (technical, financial, or simply generic risk situations). The second one looks instead at the availability of a security capital that can be sufficient to face unexpected losses, and overcome possible crises.

The insurance provides financial safety against unlikely losses related to specific events that may occur to a contractor. Through the principle of the law of large numbers and the grouping of risks, the insurance distributes the negative financial effects of these losses on a pool of similar risks over time. Since the future is uncertain, the uncertainty of the loss persists both for the policyholder (to the extent that the risk is not insured) and for the insurer and this probable loss 
is due to both the risk and the uncertainty.

The risk and the uncertainty are, in fact, fundamental for the functioning of the insurance and their sources must be understood and recognized in order to properly perform the risk management function by an insurer. It is important to distinguish between the concepts of risk and that of uncertainty, since the risk is the effect of actual results that differ from the expected results due to the stochastic nature of the same, instead the uncertainty represents a non-measurable or unknowable variation of the results. The goal is not to eliminate risks and uncertainties, as they are inherent in the insurance business, but to manage them in a financially sound manner, through appropriate techniques such as the risk sharing, the benefits, and so on. The risk and the uncertainty associated with a portfolio of policies or a company/group can derive from multiple sources. These sources may or may not be correlated or interdependent. Their relationships create dependency uncertainty, which may differ depending on the situation (for example, the correlation between sources in situations such as credit default and policyholder maturities can significantly increase in adverse conditions such as a recession).

These sources of risk and uncertainty differ in terms of coverage, market, distribution system and jurisdiction. Some may change each year, as external conditions or internal decisions can change rapidly; others, like mortality, tend to change slowly. The portfolio values generally depend on a large number of different sources, known as risk factors, so that two different portfolios characterized by the same risk measure may behave very differently due to the same market variations. Therefore, it is important to integrate the calculation of a risk measure with the calculation of its risk contributions. Because, due to diversification, the most useful risk measures are not additive, namely the sum of the risk of each part is different from the risk of the whole, dividing a risk measure into separate contributions is generally a non-trivial task. In this regard, it is important the contribution of Frees [1], which deals with the issue of stochastic life contingencies.

\section{Research Aims}

For the risk management, the insurance companies use many techniques for which application it is need to identify the various risk factors and, among these, both economic factors (such as investment returns, inflation, unemployment and increases in wages) and demographic factors (such as mortality, fertility, disability, immigration, marriage rates and retirement rates).

The identification of the risk factors takes place through the decomposition of the sources of uncertainty, decomposition which makes it possible to understand the importance of each source and to quantify its contribution to determine the overall risk. Some approaches have used techniques borrowed from valuation theories typically adopted in finance, whose application requires an axiomatic apparatus that is difficult to accept in the insurance field (Gerber [2], Zhang and 
Rachev [3], Marchioro [4]).

The most reasonable approach consists, however, in the generalization of the classical models of actuarial mathematics in which to incorporate also the financial aspect. The classic actuarial models for the life insurance limit, in fact, the randomness to the demographic sphere, that is to the demographic risk that represents every risk caused by the possible random durations of the life of the insurers (Gründl et al. [5]).

The present paper, instead, on the other hand, expands the horizon and also considers the financial or the investment risk, namely the one caused by facts concerning the market in which the insurer makes investments relating to the mathematical reserves and the own capital. The objective of the paper is, in fact, to identify and to analyze some "causes" that give rise to the risks present in the insurance field with the aim to describe some sources of uncertainty of the insurance systems, sources that feed the global risk: the decomposition of these sources allow to understand the importance of each source and to determine its contribution.

Traditionally, the importance of each source of risk is estimated using the sensitivity analysis (Norberg [6]), which consists in slightly modifying the values assumed by the parameters of the system and in observing the consequent variations. The academicians have concentrated above all on the sensitivity analysis related to life insurance contracts (Christiansen [7]). In this paper, instead, in order to assess the relative importance of each source of risk, some financial risk assessment tools are used and analyzed in detail, relating to the management of a portfolio of life insurances policies, such as the determination coefficient and the portfolio risk index. These tools represent measures to decompose the risk; their study allows to evaluate the effectiveness of the different risk management techniques and, in particular, both to understand the importance of each risk source and to quantify the single contribution for the determination of the overall risk.

In reality, on the assessment of the risk, a topic of particular relevance, numerous proposals have been put forward and the literature developed on this interesting topic has led to new approaches that make it possible to assess the overall risk, decomposing it into components attributable to the main sources of risk.

Very important are, for example, the statistical risk decomposition techniques. The most classic of the latter is the decomposition of the variance, proposed by Bühlmann [8], Fischer [9], Martin and Tasche [10], Christiansen and Helwich [11]. More recent alternatives are the Hoeffding's decomposition (Rosen and Saunders [12]) and the decomposition through the Taylor's expansion, (Christiansen [13]).

The new methodology suggested by Karabey et al. [14] is based on, instead, both the decomposition of the variance and the decomposition of Hoeffding and the expansion of Taylor. Technical insights those ones by: Gahin [15], Dickinson [16], Gatzert et al. [17], Altuntas et al. [18], Hoem [19] and Kling [20].

Schilling et al. [21] also propose a risk decomposition of an insurance portfo- 
lio holding liability policies assuming that all risk factors are a function of a state process driven by a d-dimensional Brownian motion and they obtain the final formula both using Malliavin derivatives and the conditional expectations derivatives as proposed by Markov.

In the current manuscript, instead, as a tool for assessing the relative importance of insurance systems models, a new approach is described for the decomposition of uncertainty in different sources. The decomposition of the sources of uncertainty that feed the global risk is used, and this makes it possible to understand the importance of each source and to determine its contribution.

This manuscript is based on a statistical approach too. More precisely, the methodological innovation of the paper is that the overall risk is decomposed considering not the variance of the portfolio return as an indicator of the risk itself, but the risk index of the latter. All this one is made without the need to define a risk function with respect to the various factors, but specifying a linear structure of the insurance portfolio return in which each component also depends on the value assigned by the market to it and without the need to make assumptions regarding the distribution of the overall risk. In addition, while in the contributions mentioned above it is generally assumed that the insurer's expectations on the yield of the portfolio determine its overall risk, in the formulation proposed by the authors it is assumed that are the different risk factors that influence the expected value of the portfolio (as this last variable appears in the denominator of the risk index).

The authors, thus, achieve a decomposition of the overall risk into easily identifiable and easily calculable factors, without limiting themselves to isolating only the life risk and the market risk or using mathematical and statistical tools that are particularly complex and difficult to interpret for practitioners, as it often happens in literature.

The paper is organized as follows. In the Section 2, after the introduction, the authors present the aim of the paper, instead in the Section 3 they introduce, formally, the problem and search to define and to describe briefly two important risk indicators, the risk index and the coefficient of determination, as a measure of risk decomposition. Moreover in the same section, through the decomposition of the risk index, the features of another important risk indicator, the risk pooling, are referenced. The Section 4, finally, provides a conclusion and a general discussion on directions for future research. In fact, given the relevance of this topic, the authors hope in the growth of the research in this area. The article ends with some concluding remarks while the last part of this article represents the reviewed references.

\section{Some Risk Indicators: The Coefficient of Determination and the Risk Index, as Measures for the Risk Decomposition}

The insurance companies use appropriate statistical and actuarial methods in order to protect themselves from future disbursements due to claims or man- 
agement costs, thus constituting the so-called technical reserves where the term technical reserves means the necessary provision that the company must have to meet the commitments undertaken towards the insured, and as such they are mainly determined by adding the claims reserve to the premium reserve. And just the technical reserves are the first measure to safeguard the solvency of the insurance companies, where the term solvency implies the availability of sufficient financial means to meet present and future commitments towards insured over a reference time frame. This occurs with a pre-established confidence level, corresponding to the probability of the percentile of the distribution of the random variable "overall value of the damage", taken as a safety target. So, without a doubt, the constant control and evaluation, as correct as possible, of the reserves is of primary importance.

In this paragraph, the authors address the problem of the risk analysis relating to a portfolio of insurance policies of an insurance company in order to decompose it and to determine the part relating to the insurance risk and that relating to the financial risk, namely they try to analyze and highlight tools to quantify the weight of the financial risk in the context of the global risk related to the management of a life insurance policies portfolio.

The starting point is the consideration of the financial (or investment) risk that represents any risk caused by facts concerning the market in which the insurer makes investments relating to the mathematical reserves and to the own capital. In particular, the authors try to describe some risk indicators such as the coefficient of determination and the risk index of the portfolio; the study of these instruments allow to evaluate the effectiveness of the various risk management techniques and serve to validate the importance to attribute to the financial component in the risk analysis. To achieve this goal the authors refer to a portfolio of life insurance policies made up of identical policies, each of which issued on a head of age $u$ and $Z(c)$ the random variable that denotes the total present value of all benefits payable in reference to the portfolio considered. The study of a portfolio of life insurance policies is chosen both for the considerable practical interest, due to the spread of these policies, and for their structure which makes the study particularly interesting from a mathematical point of view.

\subsection{The Portfolio Risk Index}

The riskiness of a policy portfolio can also be measured through the risk index and therefore it is interesting to determine its decomposition in such a way as to identify the part of it relating to the insurance risk and that relating to the financial risk.

The identification of the insurance risk has the dual purpose to study the value of the probability that the company will suffer losses beyond a certain levels in the future and to provide indications regarding the maximum sum that the company can afford, in relation to a given form of insurance, without compromising its stability. 
The risk index of the portfolio, which is indicated with $\frac{\sigma_{P}}{P}$, is, according to statistical terminology, the "coefficient of variation" of the random variable in question (so that the random variable $\frac{Z(c)}{c}$ ) and it is given by the ratio between the standard deviation and the average value, namely:

$$
\frac{\sigma_{P}}{P}=\frac{\sqrt{V\left[\frac{Z(c)}{c}\right]}}{E\left[\frac{Z(c)}{c}\right]}
$$

For simplicity of calculation we refer to the square of the risk index:

$$
\frac{\sigma_{P}^{2}}{P^{2}}=\frac{V\left[\frac{Z(c)}{c}\right]}{\left\{E\left[\frac{Z(c)}{c}\right]\right\}^{2}}
$$

The objective of the paper is to explicit the square of $\frac{\sigma_{P}}{P}$ to separate, in the context of the global risk, the financial contribution from that of the demographic nature.

Since, according to Frees [22] and Parker [23] [24]:

$$
\left\{E\left[\frac{Z(c)}{c}\right]\right\}^{2}=\frac{1}{c^{2}}\{E[Z(c)]\}^{2}=\left\{\sum_{k_{1}=1}^{\omega-u-1} P_{u} E\left[\mathrm{e}^{-\gamma\left(k_{1}\right)}\right]\right\}^{2}
$$

the (1) can be written as:

$$
\frac{\sigma_{P}^{2}}{P^{2}}=\frac{\frac{1}{c} E\left[Z_{i}^{2}\right]+\frac{(c-1)}{c} \sum_{k_{1}=1}^{\omega-u-1} \sum_{k_{2}=1}^{\omega-u-1} k_{1} p_{u} k_{2} p_{u} E\left[\mathrm{e}^{-\gamma\left(k_{1}\right)} \mathrm{e}^{-\gamma\left(k_{2}\right)}\right]-\left\{\sum_{k_{1}=1}^{\omega-u-1} k_{1} p_{u} E\left[\mathrm{e}^{-\gamma\left(k_{1}\right)}\right]\right\}^{2}}{\left\{\sum_{k_{1}=1}^{\omega-u-1} k_{1} P_{u} E\left[\mathrm{e}^{-\gamma\left(k_{1}\right)}\right]\right\}^{2}}
$$

which, in turn, can be rewritten as:

$$
\frac{\sigma_{P}^{2}}{P^{2}}=\frac{\frac{1}{c} E\left[Z_{i}^{2}\right]+\frac{(c-1)}{c} \sum_{k_{1}=1}^{\omega-u-1} \sum_{k_{2}=1}^{\omega-u-1} k_{1} P_{u} k_{2} P_{u} E\left[\mathrm{e}^{-\gamma\left(k_{1}\right)} \mathrm{e}^{-\gamma\left(k_{2}\right)}\right]}{\left\{\sum_{k_{1}=1}^{\omega-u-1} k_{1} P_{u} E\left[\mathrm{e}^{-\gamma\left(k_{1}\right)}\right]\right\}^{2}}-1
$$

By placing:

$$
\frac{\frac{1}{c} E\left[Z_{i}^{2}\right]+\frac{(c-1)}{c} \sum_{k_{1}=1}^{\omega-u-1} \sum_{k_{2}=1}^{\omega-u-1} k_{1} P_{u} k_{2} P_{u} E\left[\mathrm{e}^{-\gamma\left(k_{1}\right)} \mathrm{e}^{-\gamma\left(k_{2}\right)}\right]}{\left\{\sum_{k_{1}=1}^{\omega-u-1} k_{1} P_{u} E\left[\mathrm{e}^{-\gamma\left(k_{1}\right)}\right]\right\}^{2}}=\vartheta
$$

the previous expression becomes:

$$
\frac{\sigma_{P}^{2}}{P^{2}}=\vartheta-1
$$




\section{1) Formulation of the risk index in the case all insured have the same risk} profile (homogeneity of risk)

Consider a life insurance portfolio consisting of policies that provide for the collection of an annual premium by each insured and the payment of an annual compensation upon the occurrence of a specific damaging event, compensation paid from the moment in which the event in question occurs until the death of the insured person.

Suppose that all the insureds live for a maximum number of years equal to $k$ and let $t$ and $\tau$ the average life expectancy respectively of the $n_{i}$ insured individuals who pay the premiums (the so-called "payers") and of the insured individuals who receive the compensation (the so-colled "receivers"). Even for hypothesis the policies are subscribed at the initial time $i=0$ by individuals having the same age.

Let $X$ be the survival event of the generic insured, payer or receiver, for the whole period of its expected life and let $P(X)$ be the expected probability at the time $i$ that the life of the generic insured is exactly equal to its life expectancy estimated at time $i$.

Let $Z$ be the damaging event in question and $Q(Z)$ be the expected probability that the harmful event that determines the interruption of the collection of the premium by the single payer and the beginning of the payment of the compensation to the insured will not occur between the period $i$ and the period $t$.

Initially, the case in which all the insured people present the same risk respect to $X$ and $Z$ events and, consequently, a single risk class for the payers and for the receivers is considered.

Let $Y_{i}$ the value of the portfolio $P$ (considered as the price that an investor would be willing to pay to hold it) at time $i$ :

$$
Y_{i}=L_{i}+\alpha E\left(L_{i+t}\right) P(X) Q(Z)-D_{i}-\beta E\left(D_{i+\tau}\right) F(X)-b_{i} U_{i}-\varphi_{i}
$$

where:

$L_{i}$ is the incoming cash flow that the insurer receives from the payers in year $i$; $E\left(L_{i+t}\right)$ represents the expected value of the premiums that the insurer will receive from the payers for the period of their life expectancy. It is assumed that $L_{i+t}$ is discounted at the interest rate $r_{i}=0 \forall i$;

$P(X)$ is the expected survival probability of survival of the payers between the period $i$ and the period $t$;

$Q(Z)$ is the expected probability that the damaging event that determines the interruption of the payment of the premium by the single payer and the beginning of the collection of the compensation between the period $i$ and the period $t$ will not occur;

$D_{i}$ is the outgoing cash flow that the insurer pay in the year $i$ to the receivers; $E\left(D_{i+\tau}\right)$ represents the expected value of the indemnities that the insurer will have to pay to the the receivers for the period of their life expectancy. It is assumed that $D_{i+\tau}$ is discounted at the interest rate $r_{i}=0 \forall i$;

$F(X)$ is the expected survival probability of survival of the receivers between the period $i$ and the period $\tau$; 
$U_{i}$ is the outgoing cash flow incurred in the year $i$ by the insurer due to $M_{f}$ market factors to which the life annuity portfolio is exposed, namely $U_{i}=h\left(M_{i 1}, \cdots, M_{i f}\right)$.

$\phi_{i}$ is a residual term that represents the outgoing cash flow incurred by the insurer during the year $i$ due to the factors other than demographic and market factors. It is assumed that this term is uncorrelated with all the others;

$\alpha$ and $\beta$ are constants that assume values between 0 and 1 and indicate the value that a hypothetical investor would attribute to the flows $E\left(L_{i+t}\right)$ and $E\left(D_{i+\tau}\right)$ for $i=1, \cdots, k$.

$b_{i}$ is a coefficient that assumes a value between 0 and 1 and it is given by the average of the factors of exposure of the portfolio to $N$ market factors at time $i$, namely $b_{i}=\sum_{J=1}^{N} b_{i j}$ where $b_{i j}$ indicates the exposure of the portfolio with respect to the $j$-th market factor at time $i$.

Assuming that both $U_{i}$ and $b_{i}$ are distributed as normal random variables, their product is still a normal random variable which is henceforth indicated with $\delta_{i}$.

Assuming that each payer pays a premium of a fixed amount equal to $L$ and that each receiver receives a compensation equal to a fixed amount $D$, the expression (8) becomes:

$$
Y_{i}=L n_{i}+\alpha L E\left(n_{i+t}\right) P(X) Q(Z)-D m_{i}-\beta D E\left(m_{i+\tau}\right) F(X)-\delta_{i}-\varphi_{i}
$$

Suppose that $n_{i+t}$ and $m_{i+\tau}$ are distributed as normal random variables:

$$
\begin{aligned}
n_{i+t} & \sim N\left(\mu_{n_{i+t}} ; \sigma_{n_{i+t}}\right) \\
m_{i+\tau} & \sim N\left(\mu_{m_{i+t}} ; \sigma_{m_{i+t}}\right)
\end{aligned}
$$

and that $X$ and $Z$ are distributed as Bernoulli random variables:

$$
\begin{gathered}
X \sim B_{e}(P), B_{e}(F) \\
P^{x}(1-P)^{x}, \quad F^{x}(1-F)^{1-x}
\end{gathered}
$$

where:

$x$ takes the value 1 in the event of survival of the payer and of the receiver respectively up to the period $t$ and up to the period $\tau$.

$x$ takes the value 0 in the event of non-survival of the payer insured and of the receiver insured, respectively up to period $t$ and up to period $\tau$.

$$
\begin{gathered}
Z \sim B_{e}(Q) \\
Q^{z}(1-Q)^{1-z}
\end{gathered}
$$

where:

$z$ takes the value 1 in the event that the event $Z$ does not occur.

$Z$ takes the value 0 if the event $Z$ does not occur.

Then, the (9) becomes:

$$
Y_{i}=L n_{i}+\alpha L \mu_{n_{i+t}} P Q-D m_{i}-\beta D \mu_{m_{i+\tau}} F-\delta_{i}-\varphi_{i}
$$

where $\mu_{n_{i+t}}$ and $\mu_{m_{i+\tau}}$ are respectively the mean of the payers with average life 
expectancy equal to $t$ and the average of the receivers with average life expectancy equal to $\tau$.

Considering the total value of the portfolio $Y$ as the sum of the inflows and of the outflows associated with it for the $k$ life periods of the insured, the (10) becomes:

$$
\begin{aligned}
Y= & \sum_{i=1}^{k-1}\left[L n_{i}+\alpha L \mu_{n_{i+t}} P Q-D m_{i}-\beta D \mu_{m_{i+\tau}} F-\delta_{i}-\varphi_{i}\right] \\
& +L n_{k}-D m_{k}-\delta_{k}-\varphi_{k}
\end{aligned}
$$

Considering $X$ and $Z$ as two independent random variables, they can be represented according to a Bernoulli process. Regarding the $X$ event there are $X_{1}, \cdots, X_{i}, \cdots, X_{k-1}$ attempts and the number of successes, (namely the number of times that $X_{i}$ takes value 1 ), after $k-1$ attempts performed, $S_{X}$, is given by the random variable:

$$
S_{X}=X_{1}+\cdots+X_{k-1}
$$

from which:

$$
\begin{aligned}
& P\left(S_{X}=\varepsilon\right)=\left(\begin{array}{c}
k-1 \\
\varepsilon
\end{array}\right) P^{\varepsilon}(1-P)^{k-1-\varepsilon} \\
& F\left(S_{X}=\lambda\right)=\left(\begin{array}{c}
k-1 \\
\lambda
\end{array}\right) F^{\lambda}(1-F)^{k-1-\lambda}
\end{aligned}
$$

where:

$P\left(S_{X}=\varepsilon\right)$ represents the probability that $\varepsilon$ cases of survival of the payers occur, multiplied by the probability that any of them will occur while $k-1-\varepsilon$ cases of death of the payers, multiplied by the probability that any of them will occur.

$F\left(S_{X}=\lambda\right)$ represents the probability that $\lambda$ cases of survival of the payers occur, multiplied by the probability that any of them will occur while $k-1-\lambda$ cases of death of the payers, multiplied by the probability that any of them will occur.

Similarly, with respect to the event $Z$ we have $Z_{1}, \cdots, Z_{i}, \cdots, Z_{k-1}$ tests and the number of successes, (namely the number of times $Z_{i}$ assumes value 1), after the $k-1$ tests performer, $S_{Z}$ is given by the random variable:

$$
\begin{gathered}
S_{Z}=Z_{1}+\cdots+Z_{k-1} \\
Q\left(S_{Z}=\eta\right)=\left(\begin{array}{c}
k-1 \\
\eta
\end{array}\right) Q^{\eta}(1-Q)^{k-1-\eta}
\end{gathered}
$$

and so the (11) becames:

$$
\begin{aligned}
Y= & \sum_{i=1}^{k-1}\left[L n_{i}+\left(\begin{array}{c}
k-1 \\
\varepsilon
\end{array}\right) P^{\varepsilon}(1-P)^{k-1-\varepsilon}\left(\begin{array}{c}
k-1 \\
\eta
\end{array}\right) Q^{\eta}(1-Q)^{k-1-\eta} \alpha L \mu_{n_{i+t}}-D m_{i}\right. \\
& \left.-\left(\begin{array}{c}
k-1 \\
\lambda
\end{array}\right) F^{\lambda}(1-F)^{k-1-\lambda} \beta D \mu_{m_{i+\tau}}-\delta_{i}-\varphi_{i}\right]+L n_{k}-D m_{k}-\delta_{k}-\varphi_{k}
\end{aligned}
$$

where $Y$ is the total value of the portfolio $P$, given by the sum of the incoming and outgoing flows in the $k$ periods considered. 
Recalling that the goal of the paper is to explicit the square of $\frac{\sigma_{P}}{P}$ the authors proceed with the calculation of the variance of the portfolio following the approach suggested by Marchioro [25] for the determination of the standard deviation of a portfolio with a linear return structure.

As the standard deviation $\sigma_{Y}$ of the portfolio $P$ is defined as follows:

$$
\sigma_{Y}=\frac{\operatorname{Cov}(Y, Y)}{\sigma_{Y}}
$$

the (12) becomes:

$$
\begin{aligned}
\sigma_{Y}= & \frac{1}{\sigma_{Y}} \operatorname{Cov}\left[Y, \sum_{i=1}^{k-1}\left(L n_{i}+\left(\begin{array}{c}
k-1 \\
\varepsilon
\end{array}\right) P^{\varepsilon}(1-P)^{k-1-\varepsilon}\left(\begin{array}{c}
k-1 \\
\eta
\end{array}\right) Q^{\eta}(1-Q)^{k-1-\eta} \alpha L \mu_{n_{i+t}}\right.\right. \\
& \left.\left.-D m_{i}-\left(\begin{array}{c}
k-1 \\
\lambda
\end{array}\right) F^{\lambda}(1-F)^{k-1-\lambda} \beta D \mu_{m_{i+\tau}}-\delta_{i}-\varphi_{i}\right)\right] \\
& +\frac{1}{\sigma_{Y}} \operatorname{Cov}\left\{Y, L n_{k}-D m_{k}-\delta_{k}-\varphi_{k}\right\}
\end{aligned}
$$

By the last expression, as shown in the Section I of the Appendix, it is obtained:

$$
\begin{aligned}
\frac{\sigma_{P}^{2}}{P^{2}}= & \vartheta-\frac{1}{\sigma_{Y}} L\left\{\sum_{i=1}^{k}\left[\rho_{Y, n_{i}} \sigma_{n_{i}}\right]+\alpha P\left(S_{X}\right) Q\left(S_{Z}\right) \sum_{i=1}^{k-1}\left[\rho_{Y, \mu_{n i+t}} \sigma_{\mu_{n i+t}}\right]\right\} \\
& +\frac{1}{\sigma_{Y}} D\left\{\sum_{i=1}^{k}\left[\rho_{Y, m_{i}} \sigma_{m_{i}}\right]+\beta F\left(S_{X}\right) \sum_{i=1}^{k-1}\left[\rho_{Y, \mu_{m i+\tau}} \sigma_{\mu_{m i+\tau}}\right]\right\} \\
& +\frac{1}{\sigma_{Y}} \sum_{i=1}^{k}\left[\rho_{Y, \delta_{i}} \sigma_{\delta_{i}}\right]+\frac{1}{\sigma_{Y}} \sum_{i=1}^{k}\left[\sigma_{\varphi_{i}}^{2}\right]
\end{aligned}
$$

This is the formulation of the risk index in the event that all the insured individuals have the same risk profile (risk homogeneity).

In this expression we can distinguish five different components of the risk index.

The first component, $\vartheta$, represents that non-diversifiable part of the portfolio risk.

The second has a negative sign, so that it tends to reduce the total risk of the portfolio. It is a function of the premiums that the insurer receives $(L)$ from the payers and depends on:

The correlation between the value of the portfolio and the number of the payers alive at the time $i: \rho_{Y, n_{i}}$;

The standard deviation of the number of the payers living at the time $i: \sigma_{n_{i}}$;

The probability of the survival of the payers in the period $i+t: P\left(S_{X}\right)$;

The probability that in the period $i+t$ the accident does not occur: $Q\left(S_{Z}\right)$;

The value that the generic investor gives to the incoming cash flows at the period $i: \alpha$;

The correlation between the value of the portfolio and the expected value of the number of the payers in the period $i+t: \rho_{Y, \mu_{n i+t}}$;

The standard deviation of the number of the payers living in the period $i+t$ : $\sigma_{\mu_{n i+t}} ;$ 
The third component, that represents the demographic risk and positively contributes to the overall risk, is a function of the payments that the insurer makes towards the receivers $(D)$ and depends on:

The correlation between the value of the portfolio and the number of receivers living at the time $i: \rho_{Y, m_{i}}$;

The standard deviation of the number of the receivers living at the time $i$ : $\sigma_{m_{i}}$;

The probability of survival of the receivers in the period $i+\tau: F\left(S_{X}\right)$;

The value that the generic investor gives to the cash outflows in the period $i: \beta$;

The correlation between the value of the portfolio and the number of receivers alive in the period $i+\tau: \rho_{Y, \mu_{m i+\tau}}$;

The standard deviation of the number of the receivers living in the period $i+\tau: \sigma_{\mu_{m i+\tau}}$;

The fourth component expresses the contribution of the total risk due to market factors that influence the value of the portfolio, namely it indicates the market risk. It depends on:

The correlation between the value of the portfolio and the variable that expresses the expenditure due to manifestations of the market risks to which the portfolio is exposed at the time $i: \rho_{Y, \delta_{i}}$;

From the standard deviation of the latter variable: $\sigma_{\delta_{i}}$;

The fifth component is the idiosyncratic risk of the portfolio.

2) Formulation of the risk index in the case of subdivision of the insured into risk classes (inhomogeneity of the risk)

Consider now the case in which the payers and receivers belong to different $J$ classes of risk. The premium paid by the payer and the compensation received by the receiver depends on the risk class to which he belongs.

Under this hypothesis, the (9) becomes:

$$
\begin{aligned}
& Y=\sum_{j=1}^{J} \sum_{i=1}^{k-1}\left[\left(L_{j} n_{i j}+\left(\begin{array}{c}
k-2 \\
\varepsilon
\end{array}\right) P_{j}^{\varepsilon}\left(1-P_{j}\right)^{k-2-\varepsilon}\left(\begin{array}{c}
k-2 \\
\eta
\end{array}\right) Q_{j}^{\eta}\left(1-Q_{j}\right)^{k-2-\eta} \alpha L_{j} \mu_{n_{i j+t}}\right.\right. \\
& \left.-D_{j} m_{i j}-\left(\begin{array}{c}
k-2 \\
\lambda
\end{array}\right) F_{j}^{\lambda}\left(1-F_{j}\right)^{k-2-\lambda} \beta D_{j} \mu_{m_{i j+\tau}}-\delta_{i}-\varphi_{i}\right) \\
& \left.+L_{j} n_{j, k}-D_{j} m_{j, k}-\delta_{k}-\varphi_{k}\right]
\end{aligned}
$$

that is the equivalent of the (12) in the case of inhomogeneity of the risk. The

(15) for simplicity of notation is rewritten as:

$$
\begin{aligned}
Y= & \sum_{j=1}^{J} \sum_{i=1}^{k-1}\left[\left(L_{j} n_{i j}+P_{j}\left(S_{X}\right) Q_{j}\left(S_{Z}\right) \alpha L_{j} \mu_{n_{i j+t}}-D_{j} m_{i j}\right.\right. \\
& \left.\left.-F_{j}\left(S_{X}\right) \beta D_{j} \mu_{m_{i j+\tau}}-\delta_{i}-\varphi_{i}\right)+L_{j} n_{j, k}-D_{j} m_{j, k}-\delta_{k}-\varphi_{k}\right]
\end{aligned}
$$

The portfolio standard deviation is computed, again, as:

$$
\sigma_{Y}=\frac{\operatorname{Cov}(Y, Y)}{\sigma_{Y}}
$$


from which:

$$
\begin{aligned}
\sigma_{Y}= & \frac{1}{\sigma_{Y}} \operatorname{Cov}\left\{\sum_{j=1}^{J} \sum_{i=1}^{k-1} Y,\left[L_{j} n_{i j}+\left(\begin{array}{c}
k-1 \\
\varepsilon
\end{array}\right) P_{j}^{\varepsilon}\left(1-P_{j}\right)^{k-1-\varepsilon}\left(\begin{array}{c}
k-2 \\
\eta
\end{array}\right) Q_{j}^{\eta}\left(1-Q_{j}\right)^{k-1-\eta} \alpha L_{j} \mu_{n_{i j+t}}\right.\right. \\
& \left.-D_{j} m_{i j}-\left(\begin{array}{c}
k-1 \\
\lambda
\end{array}\right) F_{j}^{\lambda}\left(1-F_{j}\right)^{k-1-\lambda} \beta_{i} D_{j} \mu_{m_{i j+\tau}}-\delta_{i}-\varphi_{i}\right] \\
& \left.+\operatorname{Cov}\left(Y, L_{j} n_{j, k}-D_{j} m_{j, k}-\delta_{k}-\varphi_{k}\right)\right\}
\end{aligned}
$$

by the (17), as demonstrated in the Section II of the Appendix, it is obtained:

$$
\begin{aligned}
\frac{\sigma_{P}^{2}}{P^{2}}= & \vartheta-\frac{1}{\sigma_{Y}} \sum_{j=1}^{J} L_{j}\left\{\sum_{i=1}^{k}\left[\rho_{Y, n_{i j}} \sigma_{n_{i j}}\right]+\alpha P_{j}\left(S_{X}\right) Q_{j}\left(S_{Z}\right) \sum_{i=1}^{k-1}\left[\rho_{Y, \mu_{n_{i j+t}}} \sigma_{\mu_{n_{i j+t}}}\right]\right\} \\
& +\frac{1}{\sigma_{Y}} \sum_{j=1}^{J} D_{j}\left\{\sum_{i=1}^{k}\left[\rho_{Y, m_{i j}} \sigma_{m_{i j}}\right]+\beta F_{j}\left(S_{X}\right) \sum_{i=1}^{k-1}\left[\rho_{Y, \mu_{m_{i j+\tau}}} \sigma_{\mu_{m_{i j+\tau}}}\right]\right\} \\
& +\frac{1}{\sigma_{Y}} \sum_{i=1}^{k}\left[\rho_{Y, \delta_{i}} \sigma_{\delta_{i}}\right]+\frac{1}{\sigma_{Y}^{2}} \sum_{i=1}^{k}\left[\sigma_{\varphi_{i}}^{2}\right]
\end{aligned}
$$

This is therefore the formulation of the risk index in the case of inhomogeneity of the risk.

It is similar to (14) with the only exception that in this second case the second and the third components of the total riskiness depend on the class of risk to which the payers and the receivers belong.

\subsection{The Coefficient of Determination as Another Measure of Risk Decomposition}

The coefficient of determination can be interpreted as the proportion of uncertainty attributable to a specific source.

It is a well-known measure developed in a linear system regression analysis but it is also useful as a measure of risk attribution in a nonlinear system of insurance risks.

This approach not only justifies the measure but also provides a framework to develop competing risk attribution measures.

Indicated with $\operatorname{Var}\left[E\left(\frac{Z}{X}\right)\right]$ the measure of riskiness (of uncertainty) of the random variable $Z$ attributable to the source of uncertainty $A$, the ratio:

$$
\rho_{A Z}^{2}=\frac{V\left[E\left(\frac{Z}{A}\right)\right]}{V(A)}
$$

is defined coefficient of determination and represents the risk portion of the random variable $Z$ attributable to the source of uncertainty $A$.

With reference to a portfolio of life insurance policies, the determination coefficient is used to quantify the financial risk and its formulation as a measure of financial risk decomposition is coherent with the "risk premium" function defined by Arrow-Pratt. 
The ratio:

$$
\frac{\pi\left[E\left(\frac{Z}{A}\right)\right]}{\pi(Z)}
$$

measures the relative importance of the effect of $A$ on $Z$.

Consequently, using the approximation:

$$
\pi=\frac{r(W)}{2} V(Z)
$$

where:

$$
r(W)=-\frac{U^{\prime \prime}(W)}{U^{\prime}(W)}
$$

is the risk aversion function and $U$ is a concave utility function, the previous ratio becomes:

$$
\frac{\pi\left[E\left(\frac{Z}{A}\right)\right]}{\pi(Z)} \approx \frac{\left[r\left(\frac{W}{2}\right) V\left[E\left(\frac{Z}{A}\right)\right]\right]}{\left[\frac{r(W)}{2}\right] V(Z)} \approx \frac{V\left[E\left(\frac{Z}{A}\right)\right]}{V(Z)} \approx \rho_{A Z}^{2}
$$

that is the coefficient of determination.

This determination coefficient could be expressed in a form such as to determine the portion of risk due to the financial component (investment risk).

The coefficient of determination of the portfolio $P$ may be rewritten, with value $Y$ in relation to the market risk $M$,as follows:

$$
\rho_{Y, M}^{2}=\frac{\operatorname{Cov}\left(Y, \delta_{i}\right)}{\sigma_{Y}^{2}}=\frac{b_{i} \operatorname{Cov}\left(Y, \delta_{i}\right)}{\sigma_{Y}^{2}}
$$

where it has been assumed that the source of uncertainty $M$ is given by the outflows caused by the market factors to which the insurance portfolio is exposed. The previous expression can be rewritten as follows:

$$
\rho_{Y, M}^{2}=\frac{\rho_{Y, \delta_{i}} \sigma_{Y} \sigma_{\delta_{i}}}{\sigma_{Y}^{2}}=\frac{\rho_{Y, \delta_{i}} \sigma_{\delta_{i}}}{\sigma_{Y}}
$$

For the (23), it is:

$$
\rho_{Y, M}^{2}=\frac{\rho_{Y, \delta_{i}} \sigma_{\delta_{i}}}{\sigma_{Y}} \approx \frac{\pi\left[E\left(\frac{Y}{M}\right)\right]}{\pi(Y)}
$$

This expression indicates that the risk aversion coefficient of Arrow-Pratt is directly proportional to the correlation between the value of the portfolio and the value of the flows paid for the exposure to the market factors, with respect to the standard deviation of these flows and with respect to the coefficient of exposure to the market factors, while it is inversely proportional to the standard deviation of the value of the portfolio.

The coefficient of determination of the portfolio $P$ may be rewritten, with 
value $Y$ in relation to the demographic risk $H$, as follows:

$$
\begin{aligned}
\rho_{Y, H}^{2}= & \frac{D \rho_{Y, D_{m_{i}}} \sigma_{Y} \sigma_{D_{m_{i}}}+F\left(S_{X}\right) \beta_{i} D \rho_{Y, \mu_{m_{i+\tau}}} \sigma_{Y} \sigma_{\mu_{m_{i+\tau}}}}{\sigma_{Y}^{2}} \\
= & \frac{D \rho_{Y, D_{m_{i}}} \sigma_{D_{m_{i}}}+F\left(S_{X}\right) \beta_{i} D \rho_{Y, \mu_{m_{i+\tau}}} \sigma_{\mu_{m_{i+\tau}}}}{\sigma_{Y}}
\end{aligned}
$$

for the (23), it is:

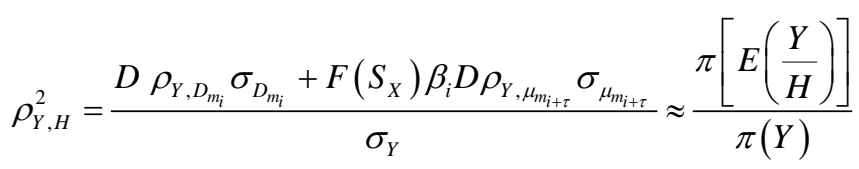

which shows that the Arrow-Pratt risk aversion index is positively dependent on the correlation of the value of the portfolio with the outflows for the payment of the indemnities at time $i$ and at time $i+1$, from the standard deviations of the same flows and from the survival probability of the insured, while it depends negatively on the standard deviation of the value of the portfolio.

From the (26) and the (28) it follows:

$$
\rho_{Y, H}^{2}=\frac{\pi\left[E\left(\frac{Y}{H}\right)\right]}{\pi\left[E\left(\frac{Y}{M}\right)\right]} \rho_{Y, M}^{2}
$$

The proof of this result is exposed in the Section III of the Appendix.

It indicates that the coefficient of determination, with respect to the demographic risk, is directly proportional to the coefficient of determination, with respect to the market risk, multiplied by a constant term.

\subsection{Risk Pooling and Non-Pooling}

Another important risk management technique is the risk sharing. Insurance organizations spread the risk through pooling. The basic premise that uncertainty can be reduced through pooling or sharing is based on the fact that the uncertain events on which the risks are based are not perfectly correlated. In fact, the smaller the relationship, the more effective the pooling mechanism. The pooling mechanism is much less effective when a common disaster affects many of an organization's clients, such as an earthquake in property and accident insurance, an AIDS epidemic in health insurance or a series of junk bond defaults in the life and in the retirement insurance.

The objective of this technique is to maximize the number of risks in the portfolio in order to activate the laws of large numbers and thus to reduce the risk assumed by the insurer. This topic was well treated by Venezian [26].

The risks that can be managed through the risk pooling can be classified into two categories:

- Pooling risk, whose effect, appropriately quantified, decreases as the size of the portfolio increases, thanks to a possible compensation performed by a simultaneous action in opposite directions on the various contracts; 
- Non-pooling risk, whose effect, appropriately quantified, increases as increases the number of the contracts, given that these risks act in the same direction on all contracts.

An example of pooling risk is offered by the demographic risk of the accidental mortality gaps while the other types of risks, present in the management of a life insurance policies portfolio, are non-pooling; financial risk is, for example, is a non-pooling risk.

It is important to note that a larger portfolio size can have a favorable effect also in relation to the financial risk (non pooling). In fact, the whole number of contracts over an extremely large amount, the insurance risk tends to disappear and this implies that, with the tendency of $c$ to infinity, the only contribution to the risk remains the financial one.

The two types of risk, observing the (5) are effectively separable. Indeed, it is:

$$
\frac{\sigma_{P}^{2}}{P^{2}}=\frac{\frac{1}{c} E\left[Z_{i}^{2}\right]+\frac{(c-1)}{c} \sum_{k_{1}=1}^{\omega-u-1} \sum_{k_{2}=1}^{\omega-u-1} k_{1} P_{u} k_{2} P_{u} E\left[\mathrm{e}^{-\gamma\left(k_{1}\right)} \mathrm{e}^{-\gamma\left(k_{2}\right)}\right]}{\left\{\sum_{k_{1}=1}^{\omega-u-1} k_{1} P_{u} E\left[\mathrm{e}^{-\gamma\left(k_{1}\right)}\right]\right\}^{2}}-1
$$

with:

$$
\begin{aligned}
& -\frac{1}{C} E\left[Z_{i}^{2}\right]+\frac{(c-1)}{c} \sum_{k_{1}=1}^{\omega-u-1} \sum_{k_{2}=1}^{\omega-u-1} k_{1} P_{u} k_{2} P_{u} E\left[\mathrm{e}^{-\gamma\left(k_{1}\right)} \mathrm{e}^{-\gamma\left(k_{2}\right)}\right] \\
& \geq\left\{\sum_{k_{1}=1}^{\omega-u-1} k_{1} p_{u} E\left[\mathrm{e}^{-\gamma\left(k_{1}\right)}\right]\right\}^{2}
\end{aligned}
$$

\section{Conclusions}

This paper exposes a methodology to decompose the uncertainty in the insurance systems into several sources namely concerning the quantitative measurement of the relative importance of different sources of risk in the insurance systems. This kind of problem is important for actuaries who are interested to identify the various factors that affect the realization of the risk (typically economic and demographic factors) or better it is very important to establish the premiums and the reserves and to determine management techniques to reduce the risk. In other words, the purpose of this article is to introduce a measure that identifies the relative importance of a source of risk and that is intuitively attractive in assessing the effectiveness of the basic risk management techniques, including the risk exchange, the sharing and the financial risk management; in this way, the risk manager can decide on the appropriate risk management tool.

The methodology developed here allows to estimate the contribution of the various risk factors; in this framework, in order to quantify the relative importance of a source of uncertainty, the authors use some statistical measures, that is, the index of risk, the coefficient of determination and the risk pooling.

Based on author's results, it is always possible to decompose the total risk index of a portfolio into five distinct components: a constant, a positive component that reduces the overall risk of the portfolio, a component that represents the demographic risk, a component that expresses the complex of market risks 
and an idiosyncratic component. In the simplifying hypothesis that all the insured individuals belong to the same risk class (homogeneity of risk), the value of the portfolio depends on, therefore, mainly on the probability of the death of the insured, on the probability of the same of being victim of the harmful event established by the insurance policy, on the average life of the insured and on the overall market risks to which the insurance portfolio is exposed. Assuming that insured individuals are grouped into different risk classes (inhomogeneity of risk), the main variables that determine the total value of the portfolio are the same, but in this case the market component of the latter also depends on the classes of risk.

The authors, then, exposed a new formulation of the coefficient of determination and of the Arrow-Pratt risk premium, noting that, in correspondence of a secure wealth $W_{0}$, for the insurer the coefficient of determination computed respect to the demographic risk, is directly proportional to the coefficient of determination computed respect to the market risk multiplied by a constant.

Finally, they underlined that risk pooling allows to significantly reduce the demographic risk of the portfolio so that only the financial risk continues to represent the main source of the overall risk, starting from the basic premise that uncertainty can be reduced through pooling only if the uncertain events upon which the risks are based are not perfectly related. Indeed, the smaller the relation, more effective is the pooling mechanism.

The authors think that this result can surely be of practical interest, since it provides a concrete methodology to select the different components that contribute to the composition of the overall risk, and by evaluating their relative importance, it is possible to use opportune techniques for controlling them.

Finally, the authors hope that in the future researches will be focused on the decomposition of statistical indices able to effectively representing the overall risk of an insurance portfolio as an alternative to the traditional and consolidated techniques of decomposition of the variance, techniques that have now shown their limits.

\section{Conflicts of Interest}

The authors declare no conflicts of interest regarding the publication of this paper.

\section{Ethical Approval}

This article does not contain any studies with human participants by any of the authors.

\section{References}

[1] Frees, E. (1990) Stochastic Life Contingencies with Solvency Considerations. Transactions of Society of Actuaries, 2, 91-148. https://pdfs.semanticscholar.org/5e84/1d387fbae8141105ab4dfa5a71586012392b.pdf

[2] Gerber, H.U. (1979) An Introduction to Mathematical Risk Theory. S S Huebner 
Foundation Monographs, University of Pensylvama Waters H, 37-43. https://doi.org/10.1080/03461238.1979.10413708

[3] Zhang, Y. and Rachev, S. (2004) Risk Attribution and Portfolio Performance Measurement: An Overview.

https://statistik.econ.kit.edu/download/doc_secure1/reviewOct3.pdf

[4] Marchioro, M. (2011) Relative Portfolio Risk Decomposition and Attribution. https://www.marchioro.org/wp-content/uploads/relative-risk-decomposition.pdf

[5] Gründl, H., Post, T. and Schulze, T. (2006) To Hedge or Not to Hedge: Managing Demographic Risk in Life Insurance Companies. The Journal of Risk and Insurance, 73, 19-41. https://doi.org/10.1111/j.1539-6975.2006.00164.x

[6] Norberg, R. (2002) Sensitivity Analysis in Insurance and Finance. Information Processes, 2, 240-242.

[7] Christiansen, M. (2008) A Sensitivity Analysis of Typical Life Insurance Contracts with Respect to the Technical Basis. Insurance: Mathematics and Economics, 42, 787-796. https://doi.org/10.1016/j.insmatheco.2007.08.005

[8] Bühlmann, H. (1995) Life Insurance with Stochastic Interest Rates. Financial Risk in Insurance, 1-24. https://doi.org/10.1007/978-3-642-57846-5_1

[9] Fischer, T. (2004) On the Decomposition of Risk in Life Insurance. https://www.researchgate.net/publication/228563299_On_the_decomposition_of_ri sk_in_life_insurance

[10] Martin, R. and Tasche, D. (2007) Shortfall: A Tail of Two Parts. Risk, 2, 84-89.

[11] Christiansen, M. and Helwich, M. (2008) Some Further Ideas Concerning the Interaction between Insurance and Investment Risks. Blätter der DGVFM, 29, 253-266. https://doi.org/10.1007/s11857-008-0053-3

[12] Rosen, D. and Saunders, D. (2010) Risk Factor Contributions in Portfolio Credit Risk Models. Journal of Banking and Finance, 34, 336-349. https://doi.org/10.1016/j.jbankfin.2009.08.002

[13] Christiansen, M. (2007) A Joint Analysis of Financial and Biometrical Risks in Life Insurance. Ph.D. Dissertation, Rostock University, Rostock.

[14] Karabey, U., Kleinow, T. and Cairns, A.J. (2014) Factor Risk Quantification in Annuity Models. Insurance: Mathematics and Economics, 58, 34-45. https://doi.org/10.1016/j.insmatheco.2014.06.004

[15] Gahin, F. (1971) Review of the Literature on Risk Management. The Journal of Risk and Insurance, 38, 309-313. https://doi.org/10.2307/251507

[16] Dickinson, G. (2001) Enterprise Risk Management: Its Origins and Conceptual Foundation. The Geneva Papers on Risk and Insurance, 26, 360-366. https://doi.org/10.1111/1468-0440.00121

[17] Gatzert, N., Schmeiser, H. and Schuckmann, S. (2008) Enterprise Risk Management in Financial Groups: Analysis of Risk Concentration and Default Risk. Financial Markets and Portfolio Management, 22, 241-258. https://doi.org/10.1007/s11408-008-0081-y

[18] Altuntas, M., Berry-Stölzle, T.R. and Hoyt, R. (2011) Implementation of Enterprise Risk Management: Evidence from the German Property-Liability Insurance Industry. The Geneva Papers on Risk and Insurance-Issues and Practice, 36, 414-439. https://doi.org/10.1057/gpp.2011.11

[19] Hoem, J.M. (1988) The Versatility of the Markov Chain as a Tool in the Mathematics of Life Insurance.

https://www.researchgate.net/publication/248058482_The_Versatility_of_the_Mark ov_Chain_as_a_Tool_in_the_Mathematics_of_Life_Insurance 
[20] Kling, A., Rub, J. and Schilling, K. (2014) Risk Analysis of Annuity Conversion Options in a Stochastic Mortality Environment. Astin Bullettin, 44, 197-236. https://doi.org/10.1017/asb.2014.7

[21] Schilling, K., Bauer, D., Christiansen, M. and Kling, A. (2015) Decomposing Life Insurance Liabilities into Risk Factors. Banque de France Working Papers Series, 1, $2-48$.

https://acpr.banque-france.fr/sites/default/files/medias/documents/1501_sbck-2015.pdf

[22] Frees, E.W. (2013) Relative Importance of Risk Sources in Insurance Systems. North American Actuarial Journal, 2, 34-49. https://doi.org/10.1080/10920277.1998.10595694

[23] Parker, G. (1994) Moments of the Present Value of a Portfolio of Policies. Scandinavian Actuarial Journal, 1, 53-67.

https://doi.org/10.1080/03461238.1994.10413929

[24] Parker, G. (1997) Stochastic Analysis of the Interaction between Investment and Insurance Risks. North American Actuarial Journal, 1, 55-84. https://doi.org/10.1080/10920277.1997.10595604

[25] Marchioro, M. (2017) A Risk Decomposition Framework Consistent with Performance Measurements.

https://www.marchioro.org/papers/risk-measure-components/

[26] Venezian, C.E. (1984) Efficiency and Equity in Insurance. The Journal of Risk and Insurance, 51, 190-204. https://doi.org/10.2307/252553 


\section{Appendix}

\section{Section I}

The expression (13), reported below:

$$
\begin{aligned}
\sigma_{Y}= & \frac{1}{\sigma_{Y}} \operatorname{Cov}\left[Y, \sum_{i=1}^{k-1}\left(L n_{i}+\left(\begin{array}{c}
k-1 \\
\varepsilon
\end{array}\right) P^{\varepsilon}(1-P)^{k-1-\varepsilon}\left(\begin{array}{c}
k-1 \\
\eta
\end{array}\right) Q^{\eta}(1-Q)^{k-1-\eta} \alpha L \mu_{n_{i+t}}\right.\right. \\
& \left.\left.-D m_{i}-\left(\begin{array}{c}
k-1 \\
\lambda
\end{array}\right) F^{\lambda}(1-F)^{k-1-\lambda} \beta D \mu_{m_{i+\tau}}-\delta_{i}-\varphi_{i}\right)\right] \\
& +\frac{1}{\sigma_{Y}} \operatorname{Cov}\left\{Y, L n_{k}-D m_{k}-\delta_{k}-\varphi_{k}\right\}
\end{aligned}
$$

for simplicity of notation is rewritten as:

$$
\begin{aligned}
\sigma_{Y}= & \frac{1}{\sigma_{Y}} \sum_{i=1}^{k-1}\left[L \operatorname{Cov}\left(Y, n_{i}\right)+P\left(S_{X}\right) Q\left(S_{Z}\right) \alpha L \operatorname{Cov}\left(Y, \mu_{n i+t}\right)-\operatorname{DCov}\left(Y, m_{i}\right)\right. \\
& \left.-F\left(S_{X}\right) \beta \operatorname{DCov}\left(Y, \mu_{m i+\tau}\right)-\operatorname{Cov}\left(Y, \delta_{i}\right)-\operatorname{Cov}\left(Y, \varphi_{i}\right)\right] \\
& +\frac{1}{\sigma_{Y}}\left[\operatorname{LCov}\left(Y, n_{k}\right)-\operatorname{DCov}\left(Y, m_{k}\right)-\operatorname{Cov}\left(Y, \delta_{k}\right)-\operatorname{Cov}\left(Y, \varphi_{k}\right)\right]
\end{aligned}
$$

for the dissociative property of the summation it is obtained:

$$
\begin{aligned}
\sigma_{Y}= & \frac{1}{\sigma_{Y}} L \sum_{i=1}^{k-1}\left[\operatorname{Cov}\left(Y, n_{i}\right)\right]+\frac{1}{\sigma_{Y}} \alpha L P\left(S_{X}\right) Q\left(S_{Z}\right) \sum_{i=1}^{k-1}\left[\operatorname{Cov}\left(Y, \mu_{n i+t}\right)\right] \\
& -\frac{1}{\sigma_{Y}} D \sum_{i=1}^{k-1}\left[\operatorname{Cov}\left(Y, m_{i}\right)\right]-\frac{1}{\sigma_{Y}} \beta F\left(S_{X}\right) D \sum_{i=1}^{k-1}\left[\operatorname{Cov}\left(Y, \mu_{m i+\tau}\right)\right] \\
& -\frac{1}{\sigma_{Y}} \sum_{i=1}^{k-1}\left[\operatorname{Cov}\left(Y, \delta_{i}\right)\right]-\frac{1}{\sigma_{Y}} \sum_{i=1}^{k-1}\left[\operatorname{Cov}\left(Y, \varphi_{i}\right)\right]+\frac{1}{\sigma_{Y}} L \operatorname{Cov}\left(Y, n_{k}\right) \\
& -\frac{1}{\sigma_{Y}} \operatorname{DCov}\left(Y, m_{k}\right)-\frac{1}{\sigma_{Y}} \operatorname{Cov}\left(Y, \delta_{k}\right)-\frac{1}{\sigma_{Y}} \operatorname{Cov}\left(Y, \varphi_{k}\right)
\end{aligned}
$$

from which:

$$
\begin{aligned}
\sigma_{Y}= & \frac{1}{\sigma_{Y}} L \sum_{i=1}^{k}\left[\operatorname{Cov}\left(Y, n_{i}\right)\right]+\frac{1}{\sigma_{Y}} \alpha \operatorname{LP}\left(S_{X}\right) Q\left(S_{Z}\right) \sum_{i=1}^{k}\left[\operatorname{Cov}\left(Y, \mu_{n i+t}\right)\right] \\
& -\frac{1}{\sigma_{Y}} D \sum_{i=1}^{k}\left[\operatorname{Cov}\left(Y, m_{i}\right)\right]-\frac{1}{\sigma_{Y}} \beta F\left(S_{X}\right) D \sum_{i=1}^{k}\left[\operatorname{Cov}\left(Y, \mu_{m i+\tau}\right)\right] \\
& -\frac{1}{\sigma_{Y}} \sum_{i=1}^{k}\left[\operatorname{Cov}\left(Y, \delta_{i}\right)\right]-\frac{1}{\sigma_{Y}} \sum_{i=1}^{k}\left[\operatorname{Cov}\left(Y, \varphi_{i}\right)\right]
\end{aligned}
$$

as given two generic random variable $A$ and $B$ is worth:

$$
\operatorname{Cov}(A, B)=\rho_{A, B} \sigma_{A} \sigma_{B}
$$

and as for hypotesis the term $\varphi_{i}$ is uncorrelated with all others:

$$
\begin{aligned}
\sigma_{Y}= & \frac{1}{\sigma_{Y}} L \sum_{i=1}^{k}\left[\rho_{Y, n_{i}} \sigma_{Y} \sigma_{n_{i}}\right]+\frac{1}{\sigma_{Y}} \alpha L P\left(S_{X}\right) Q\left(S_{Z}\right) \sum_{i=1}^{k-1}\left[\rho_{Y, \mu_{n i+t}} \sigma_{Y} \sigma_{\mu_{n i+t}}\right] \\
& -\frac{1}{\sigma_{Y}} D \sum_{i=1}^{k}\left[\rho_{Y, m_{i}} \sigma_{Y} \sigma_{m_{i}}\right]-\frac{1}{\sigma_{Y}} \beta D F\left(S_{X}\right) \sum_{i=1}^{k-1}\left[\rho_{Y, \mu_{m i+\tau}} \sigma_{Y} \sigma_{\mu_{m i+\tau}}\right] \\
& -\frac{1}{\sigma_{Y}} \sum_{i=1}^{k}\left[\rho_{Y, \delta_{i}} \sigma_{Y} \sigma_{\delta_{i}}\right]-\frac{1}{\sigma_{Y}} \sum_{i=1}^{k}\left[\sigma_{\varphi_{i}}^{2}\right]
\end{aligned}
$$


by dividing both right hand side and left hand side for the term $\sigma_{Y}$ :

$$
\begin{aligned}
& \frac{1}{\sigma_{Y}} L \sum_{i=1}^{k}\left[\rho_{Y, n_{i}} \sigma_{n_{i}}\right]+\frac{1}{\sigma_{Y}} \alpha L P\left(S_{X}\right) Q\left(S_{Z}\right) \sum_{i=1}^{k-1}\left[\rho_{Y, \mu_{n i+t}} \sigma_{\mu_{n i+t}}\right] \\
& -\frac{1}{\sigma_{Y}} D \sum_{i=1}^{k}\left[\rho_{Y, m_{i}} \sigma_{m_{i}}\right]-\frac{1}{\sigma_{Y}} \beta D F\left(S_{X}\right) \sum_{i=1}^{k-1}\left[\rho_{Y, \mu_{m i+\tau}} \sigma_{\mu_{m i+\tau}}\right] \\
& -\frac{1}{\sigma_{Y}} \sum_{i=1}^{k}\left[\rho_{Y, \delta_{i}} \sigma_{\delta_{i}}\right]-\frac{1}{\sigma_{Y}} \sum_{i=1}^{k}\left[\sigma_{\varphi_{i}}^{2}\right]=1
\end{aligned}
$$

by replacing the previous in the (7) it is obtained the final expression of the (1) in the case risk homogeneity (namely assuming that all the insured belong to the same class of risk), that is the (14).

\section{Section II}

The expression (17), reported below:

$$
\begin{aligned}
\sigma_{Y}= & \frac{1}{\sigma_{Y}} \operatorname{Cov}\left\{\sum_{j=1}^{J} \sum_{i=1}^{k-1} Y,\left[L_{j} n_{i j}+\left(\begin{array}{c}
k-1 \\
\varepsilon
\end{array}\right) P_{j}^{\varepsilon}\left(1-P_{j}\right)^{k-1-\varepsilon}\left(\begin{array}{c}
k-2 \\
\eta
\end{array}\right) Q_{j}^{\eta}\left(1-Q_{j}\right)^{k-1-\eta} \alpha L_{j} \mu_{n_{i j+t}}\right.\right. \\
& \left.-D_{j} m_{i j}-\left(\begin{array}{c}
k-1 \\
\lambda
\end{array}\right) F_{j}^{\lambda}\left(1-F_{j}\right)^{k-1-\lambda} \beta_{i} D_{j} \mu_{m_{i j+\tau}}-\delta_{i}-\varphi_{i}\right] \\
& \left.+\operatorname{Cov}\left(Y, L_{j} n_{j, k}-D_{j} m_{j, k}-\delta_{k}-\varphi_{k}\right)\right\}
\end{aligned}
$$

for the additive property of the covariance may be rewritten as:

$$
\begin{aligned}
\sigma_{Y}= & \frac{1}{\sigma_{Y}} \sum_{j=1}^{J} \sum_{i=1}^{k-1}\left\{\operatorname{Cov}\left[Y, L_{j} n_{i j}\right]\right\}+\frac{1}{\sigma_{Y}} \sum_{j=1}^{J} \sum_{i=1}^{k-1}\left\{\operatorname{Cov}\left[Y,\left(\begin{array}{c}
k-1 \\
\varepsilon
\end{array}\right) P_{j}^{\varepsilon}\left(1-P_{j}\right)^{k-1-\varepsilon}\left(\begin{array}{c}
k-1 \\
\eta
\end{array}\right) Q_{j}^{\eta}\left(1-Q_{j}\right)^{k-1-\eta} \alpha L_{j} \mu_{n_{i j+t}}\right]\right\} \\
& -\frac{1}{\sigma_{Y}} \sum_{j=1}^{J} \sum_{i=1}^{k-1}\left\{\operatorname{Cov}\left(Y, D_{j} m_{i j}\right)\right\}-\frac{1}{\sigma_{Y}} \sum_{j=1}^{J} \sum_{i=1}^{k-1}\left\{\operatorname{Cov}\left[Y,\left(\begin{array}{c}
k-1 \\
\lambda
\end{array}\right) F_{j}^{\lambda}\left(1-F_{j}\right)^{k-1-\lambda} \beta D_{j} \mu_{m_{i j+\tau}}\right]\right\} \\
& +\frac{1}{\sigma_{Y}} \sum_{i=1}^{k-1}\left\{\operatorname{Cov}\left[Y, \delta_{i}\right]\right\}+\frac{1}{\sigma_{Y}} \sum_{i=1}^{k-1}\left\{\operatorname{Cov}\left[Y, \varphi_{i}\right]\right\} \\
& +\frac{1}{\sigma_{Y}} \sum_{j=1}^{J}\left[\operatorname{Cov}\left(Y, L_{j} n_{k, j}\right)\right]-\frac{1}{\sigma_{Y}} \sum_{j=1}^{J}\left[\operatorname{Cov}\left(Y, D_{j} m_{k, j}\right)\right] \\
& -\frac{1}{\sigma_{Y}} \operatorname{Cov}\left(Y, \delta_{k}\right)-\frac{1}{\sigma_{Y}} \operatorname{Cov}\left(Y, \varphi_{k}\right)
\end{aligned}
$$

and namely:

$$
\begin{aligned}
\sigma_{Y}= & \frac{1}{\sigma_{Y}} \sum_{j=1}^{J} \sum_{i=1}^{k}\left\{\operatorname{Cov}\left[Y, L_{j} n_{i j}\right]\right\}+\frac{1}{\sigma_{Y}} \alpha P_{j}\left(S_{X}\right) Q_{j}\left(S_{Z}\right) \sum_{j=1}^{J} \sum_{i=1}^{k-1}\left\{\operatorname{Cov}\left[Y, L_{j} \mu_{n_{i j+t}}\right]\right\} \\
& -\frac{1}{\sigma_{Y}} \sum_{j=1}^{J} \sum_{i=1}^{k}\left\{\operatorname{Cov}\left(Y, D_{j} m_{i j}\right)\right\}-\frac{1}{\sigma_{Y}} \beta F_{j}\left(S_{X}\right) \sum_{j=1}^{J} \sum_{i=1}^{k-1}\left\{\operatorname{Cov}\left[Y, D_{j} \mu_{m_{i j+\tau}}\right]\right\} \\
& +\frac{1}{\sigma_{Y}} \sum_{i=1}^{k}\left\{\operatorname{Cov}\left[Y, \delta_{i}\right]\right\}+\frac{1}{\sigma_{Y}} \sum_{i=1}^{k}\left\{\operatorname{Cov}\left[Y, \varphi_{i}\right]\right\}
\end{aligned}
$$

By dividing both right hand side and left hand side for the term $\sigma_{Y}$, follows: 


$$
\begin{aligned}
& \frac{1}{\sigma_{Y}} \sum_{j=1}^{J} \sum_{i=1}^{k}\left[L_{j} \rho_{Y, n_{i j}} \sigma_{n_{i j}}\right]+\frac{1}{\sigma_{Y}} \alpha P_{j}\left(S_{X}\right) Q_{j}\left(S_{Z}\right) \sum_{j=1}^{J} \sum_{i=1}^{k-1}\left[L_{j} \rho_{Y, \mu_{n_{i j+t}}} \sigma_{\mu_{n_{j j+t}}}\right] \\
& -\frac{1}{\sigma_{Y}} \sum_{j=1}^{J} \sum_{i=1}^{k}\left[D_{j} \rho_{Y, m_{i j}} \sigma_{m_{i j}}\right]-\beta F_{j}\left(S_{X}\right) \frac{1}{\sigma_{Y}} \sum_{j=1}^{J} \sum_{i=1}^{k-1}\left[D_{j} \rho_{Y, \mu_{m_{j+\tau}+\tau}} \sigma_{\mu_{m_{i j+\tau}}}\right] \\
& +\frac{1}{\sigma_{Y}} \sum_{i=1}^{k}\left[\rho_{Y, \delta_{i}} \sigma_{\delta_{i}}\right]+\frac{1}{\sigma_{Y}} \sum_{i=1}^{k}\left[\sigma_{\varphi_{i}}^{2}\right]=1
\end{aligned}
$$

Finally, by collecting the costant terms $L_{j}$ and $D_{j}$ and by replacing the resulting expression in the (7), it is obtained the final expression of the (1) in the case of Jhomogeneous risk classes, that is the (18).

\section{Section III}

From the expression (26):

$$
\rho_{Y, M}^{2}=\frac{\rho_{Y, \delta_{i}} \sigma_{\delta_{i}}}{\sigma_{Y}} \approx \frac{\pi\left[E\left(\frac{Y}{M}\right)\right]}{\pi(Y)}
$$

it is obtained:

$$
\pi(Y) \rho_{Y, M}^{2} \approx \pi\left[E\left(\frac{Y}{M}\right)\right]
$$

from which:

$$
\pi(Y) \approx \frac{\pi\left[E\left(\frac{Y}{M}\right)\right]}{\rho_{Y, M}^{2}}
$$

By replacing the previous in the (28), the authors get the (29):

$$
\rho_{Y, H}^{2} \approx \frac{\pi\left[E\left(\frac{Y}{H}\right)\right]}{\pi\left[E\left(\frac{Y}{M}\right)\right]} \rho_{Y, M}^{2}
$$

